efforts to identify criteria associated with good outcomes in this age range, literature has not found any yet. We aimed to use novel tools being reported in stroke literature, such as First Pass Effect (FPE) and Hypoperfusion Intensity Ratio (HIR), to optimize elderly patient selection for thrombectomy.

Methods A single-center retrospective analysis of a prospectively maintained large vessel occlusion database (20142019) was conducted. We included patients $\geq 80$ years old with acute ischemic stroke that underwent endovascular thrombectomy. First pass thrombectomy was defined as single attempt obtaining TICI $2 \mathrm{~b}$ or higher without distal embolization, as previously stated in literature. Collaterals were assessed based on HIR (good collaterals <0.4; poor collaterals $\geq 0.4$ ). Baseline characteristics, procedural data, and outcomes were evaluated. The association between clinical and procedural variables and outcomes at 90 days was assessed.

Results A total 146 patients were included in the study. Mean age was $87.8 \pm 4.12$ (range $80-100$ ) and $65.75 \%$ were female. Pre-stroke functional independence (baseline mRS 02) was seen in $64.1 \%$. Median time of onset to groin puncture was 261.5 minutes and mean time from groin puncture to recanalization was 37.15 minutes. Good collaterals were seen in $50.9 \%$ of patients. Successful reperfusion (TICI $2 \mathrm{~b}$ or higher) was achieved in $87.1 \%$. The rates of good outcome (mRS 0-2), acceptable outcome (mRS 0-3) and poor outcome (mRS 4-6) at 90 days were $18.5 \%, 38.1 \%$ and $43.4 \%$, respectively. Multivariate logistic regression indicated that pre-stroke functional independence $(p=0.0008)$, good collaterals $(p=0.0009)$, use of stent retriever (Solitaire, $p=0.035$; Trevo, $p=0.032)$ and balloon guide catheter $(p=0.028)$ were associated with good outcome.

Conclusions In our retrospective cohort of elderly patients treated with thrombectomy, the baseline characteristics that appear to influence in the rate of good outcome were the pre-stroke functional independence and the presence of good collaterals. Although previous studies with younger populations showed promising use of First Pass Effect measure to predict good outcome in patients who underwent thrombectomy, we did not find any significant association in patients $\geq 80$-yearold. Preference for the use of stent-retriever and balloon guide-catheter may shorten the time to reperfusion and therefore, provide better outcomes. We emphasize the need of further studies to improve selection criteria for endovascular thrombectomy in this population.

Disclosures A. Monteiro: None. G. Cortez: None. Y. Murtaza: None. A. Aghaebrahim: None. E. Sauvageau: None. R. Hanel: 2; C; Microvention, Codman, Stryker, Medtronic.

\section{E-083 A QUANTITATIVE AND QUALITATIVE ANALYSIS OF \#RADIALFIRST CONVERSATIONS ON TWITTER}

N Alotaibi*, P Harker, F Robertson, J Vranic, M Koch, C Stapleton, T Leslie-Mazwi, A Patel. Neurosurgery, Massachusetts General Hospital, BOSTON, MA

10.1136/neurintsurg-2020-SNIS.117 
Background Trans-radial access for neuro-endovascular procedures has gained popularity in recent years, driven in part by social media. The approach was adopted and modified from that used by interventional cardiology. This study analyzes Twitter conversations regarding this topic using the popular hashtag \#RadialFirst.

Materials and Methods Twitter was evaluated for public content pertaining to all trans-radial access. We conducted a mixed-method analysis that includes a quantitative examination of all social media metrics (no. of tweets, mentions, retweets, locations, word count) and a qualitative evaluation of tweets for thematic analysis.

Results Between January 2013 and February 2020, there were 100,531 tweets from 100 countries using the hashtag \#RadialFirst. These tweets had an overall impression of 218 million and were retweeted 75,000 times by 13,103 users. More than $60 \%$ of tweets carried media materials (e.g. wrist image, angiographic runs) but only $14 \%$ of tweets had a link to an article. USA (17\%) and the UK (4.2\%) had the highest numbers of tweets. Physicians authored $84 \%$ of the tweets and interventional cardiologists accounted for 94\% of the top 100 influencers for the hashtag. Tweets showed steady increase in numbers over time and coincided later with the appearance of a new hashtag for neuro-intervention, \#RadialForNeuro. Five prominent themes emerged from the coded data: sharing cases, providing operative tips, requesting information,

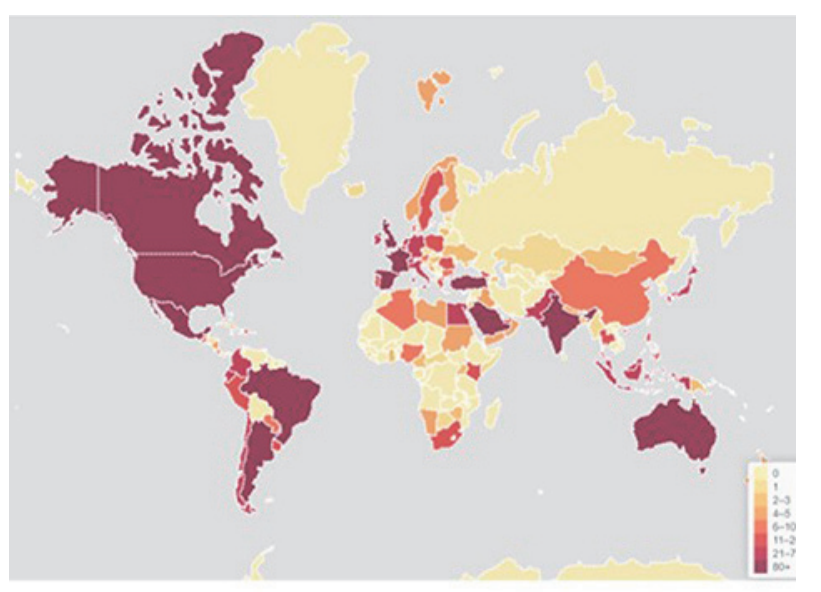

World map

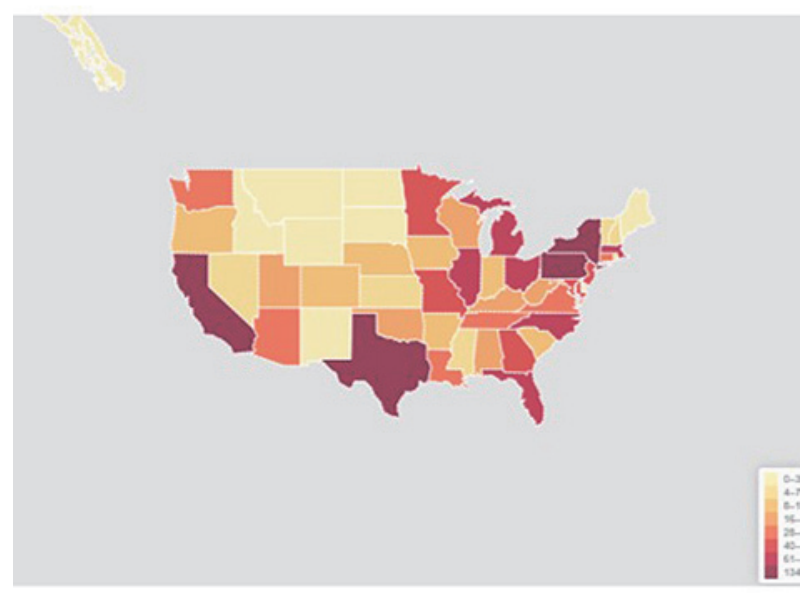

US map highlighting adverse events, and tweets related to commercial endorsements.

Conclusion This study is the first to provide insight into Twitter communications regarding trans-radial access. These findings highlight the impact of Twitter in disseminating the technique, and the role of social media in education and refinement of technical approaches.

Disclosures N. Alotaibi: None. P. Harker: None. F. Robertson: None. J. Vranic: None. M. Koch: None. C. Stapleton: None. T. Leslie-Mazwi: None. A. Patel: None.

\section{E-084 VARIABILITY IN ENDOVASCULAR TREATMENT OF INTRACRANIAL INTERNAL CAROTID ARTERY VERSUS MIDDLE CEREBRAL ARTERY OCCLUSIONS}

M Aly*, R Abdalla, M Hurley, A Shaibani, S Ansari. Intervention Radiology, Northwestern University, Chicago, IL

\subsection{6/neurintsurg-2020-SNIS. 118}

Purpose Endovascular treatment (ET) for the acute management of ischemic stroke due to large vessel occlusions is the standard of care for anterior circulation. Meta-analysis has suggested equivalent recanalization and better clinical outcomes after ET for ICA and proximal MCA occlusion (1-2). The aim of our study was to compare these two cohorts and identify any variability/bias in presentations, imaging selection, technical and clinical efficacy.

Materials and Methods This is a retrospective study of patients that underwent ET for ICA/MCA occlusions over a four-year period at three comprehensive stroke centers. We studied patient demographics, vascular risk factors, NIHSS, time of presentation, imaging, procedures details, and clinical outcomes. Chi-square, Mann-Whitney $U$ and student ttests were used for variables univariate analysis as appropriate. Multivariate analysis was performed to assess the comparability of the groups based on demographics and risk factors.

Results 185 patients were studied (mean age, $68 \pm 17$; 55\% women; median NIHSS, $16 \pm 6$ ). Both study cohorts were comparable regarding their age $(65 \pm 20$ vs $69 \pm 17$, $\mathrm{p}=0.26)$ and past medical history of Hypertension, Diabetes Mellitus, Hyperlipidemia and Smoking habit $(\mathrm{p}=0.64$, $\mathrm{p}=0.68, \mathrm{p}=0.55, \mathrm{p}=0.47$ respectively). There was no statistically significant difference between both cohorts regarding their NIHSS $(17 \pm 6$ vs $16 \pm 6, p=0.23)$ or the time from onset to groin puncture (mean 5:43:48 vs 5:24:58, $\mathrm{p}=0.54)$. Moreover, there was no significant difference between mean ASPECT $(8.3 \pm 1.3$ vs $8.6 \pm 1.3, \mathrm{p}=0.19)$ and mean CTP-rCBF $<30 \%(24.5 \mathrm{ml} \pm 36$ vs $28.3 \mathrm{ml} \pm$ $38, \mathrm{p}=0.6)$. During the procedure ICA occlusions were associated with a higher number of stent retriever passes to achieve recanalization (mean 2.5 vs $1.9, \mathrm{p}=0.028$ ), and longer time from groin puncture to recanalization (mean 1:02:11 vs 00:51:48, $\mathrm{p}=0.034)$. Also, there was a trend toward significance in the rate of successful recanalization after the first pass of stent retriever in the MCA occlusion cohort $(46 \%$ vs $33 \%, \mathrm{p}=0.09)$. However, there was no statistically significant difference regarding the rate of successful recanalization TICI $\geq 2$ b $(91 \%$ in group A vs $95 \%$ in group $\mathrm{B}, \mathrm{p}=0.35)$ or the rate of complete recanalization TICI $2 \mathrm{c} / 3$ (45\% vs $52 \%, \mathrm{p}=0.39)$. Although, the 90 -days outcome measured by mRS was not statistically significantly different 